\title{
Knowledge, attitudes and practices of primary health care physicians towards evidence-based medicine in Doha, Qatar
}

N.J. Al-Kubaisi, ${ }^{7}$ L.A. Al-Dahnaim ${ }^{7}$ and R.E. Salama ${ }^{1,2}$

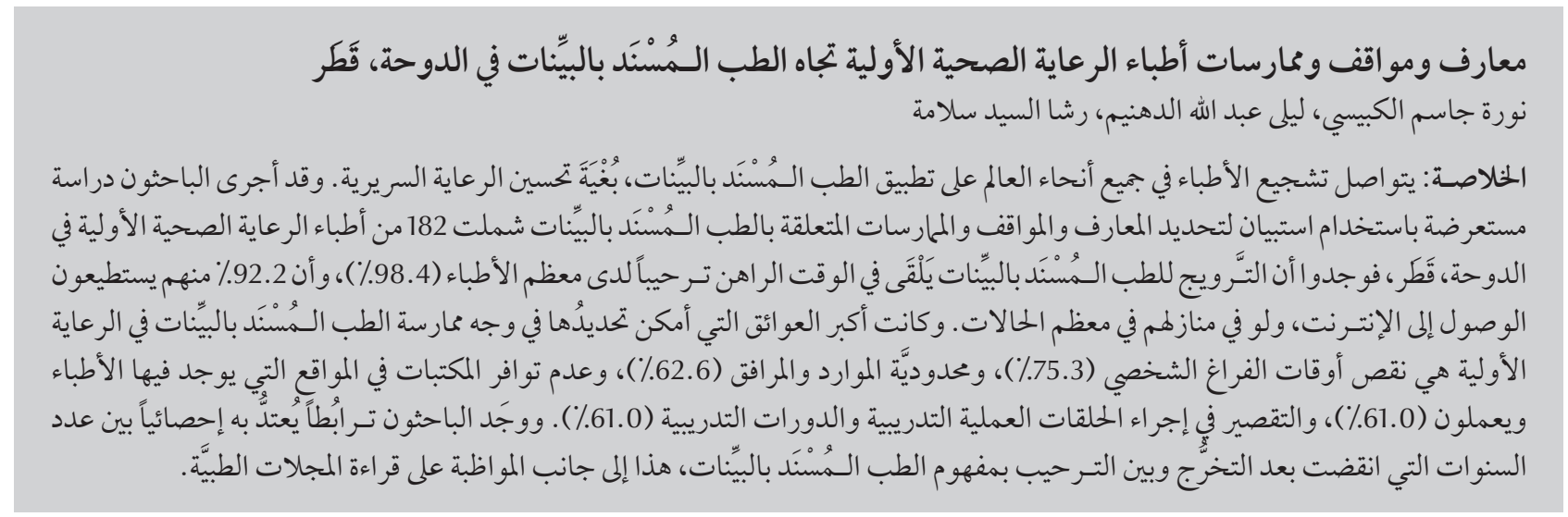

ABSTRACT Physicians worldwide are being encouraged to apply evidence-based medicine (EBM) to improve their clinical care. A cross-sectional questionnaire study was carried to determine the knowledge, attitudes and practices regarding EBM among 182 primary care physicians in Doha, Qatar. The current promotion of EBM was welcomed by most physicians (98.4\%). While $92.2 \%$ had access to the Internet, this was mostly at home. The major perceived barriers to practising EBM in primary care were lack of free personal time $(75.3 \%)$, limited resources and facilities $(62.6 \%)$, no library in the locality (61.0\%) and lack of training workshops and courses $(61.0 \%)$. There was a statistically significant association between years since graduation and welcoming the EBM concept as well as with frequency of reading journals.

Connaissances, attitudes et pratiques des praticiens de soins de santé primaires concernant la médecine fondée sur les preuves à Doha (Qatar)

RÉSUMÉ Les médecins dans le monde sont encouragés à appliquer la médecine fondée sur les preuves - ou médecine factuelle - pour améliorer leurs soins cliniques. Une étude transversale avec questionnaire a été menée pour déterminer les connaissances, les attitudes et les pratiques concernant ce type de médecine auprès de 185 médecins de soins primaires à Doha (Qatar). La promotion actuelle en faveur de la médecine factuelle a été bien accueillie par la plupart des médecins (98,4\%). Si 92,2\% d'entre eux avaient accès à Internet, c'était principalement à leur domicile. Les principaux obstacles à la pratique de la médecine factuelle ressentis dans les soins primaires étaient le manque de temps personnel $(75,3 \%)$, des ressources et infrastructures limitées (62,6\%), l'absence de bibliothèque dans la localité (61 \%) et le manque de séminaires-ateliers et cours de formation (61,0 \%). L'association entre le nombre d'années depuis l'obtention du diplôme et le bon accueil réservé au concept de médecine factuelle ainsi que la fréquence de la lecture de revues était statistiquement significative.

'Department of Community Medicine, Hamad Medical Corporation, Doha, Qatar (Correspondence to R. Salama: rashasalama2004@yahoo.com). ${ }^{2}$ Department of Community Medicine, Suez Canal University, Ismailia, Egypt.

Received: 04/02/09; accepted: 06/04/09 


\section{Introduction}

Evidence-based medicine (EBM) is the process of systematically reviewing, appraising and using clinical research findings to aid the delivery of optimum clinical care to patients [1]. The need to apply EBM and to develop EBM guidelines and performance indicators for the management of commonly encountered problems in general practice and primary care has been detailed in several papers [2-8]. Some surveys have concluded, however, that the best available evidence is seldom used when making clinical decisions [9-11].

A review of physicians' performance suggested that learning how to practise EBM, seeking out and applying the findings of EBM summaries and adopting evidence-based practice protocols developed by respected colleagues can keep us aware of medical advances and help to enhance our clinical performance [12]. EBM practice also supports decision-making shared with users, which is already favoured within the medical community as the ideal of decision-making $[13,14]$.

A pioneer study was made in the United Kingdom (UK) in 1998 to study the reaction of general practitioners (GPs) towards EBM [15]. Despite considerable variations in attitudes to the promotion of EBM, most GPs were welcoming and agreed that it improved patient care. There was a low level of awareness about extracting journals, review publications and databases relevant to EBM, and the major perceived barrier to its practice was lack of personal time. Respondents thought that the best way to move from opinionbased practice towards EBM was by using evidence-based guidelines or protocols developed by colleagues [15]. Two studies among primary health care (PHC) physicians in Saudi Arabia showed similar findings $[16,17]$. To our knowledge, no similar studies have been carried out Qatar. Therefore the aims of this study were to investigate awareness of PHC physicians in Qatar about EBM and to determine their knowledge, attitudes and practices regarding EBM. The results would help to establish the educational requirements necessary for greater use of EBM in patient care.

\section{Methods}

A cross-sectional study was carried out among physicians practising in $\mathrm{PHC}$ centres in Doha city, the capital of Qatar (population 885000 ). There are 20 primary care centres in Qatar, 11 of them located in Doha. The self-administered questionnaire was sent to all $187 \mathrm{PHC}$ physicians in Doha: 9 consultants, 41 specialists and 137 clinical associate physicians.

\section{Data collection}

We used the questionnaire adapted by Al-Ansary and Khoja for use in Saudi Arabia $[16,17]$ from McColl's original questionnaire in the UK [15]. The questionnaire has 4 parts and includes questions addressing knowledge, attitudes and practices about EBM as well as associated variables and barriers to practising it. The first part of the questionnaire includes data about the personal characteristics of the physicians: age, sex, qualifications, nationality, years since graduation and years of experience in PHC. The second part addresses practice characteristics of physicians: ever-use of resources for clinical decision-making, use of journals and understanding of medical terms. The third part asks about attitudes towards EBM: welcoming current promotion of EBM, colleagues' positive attitudes towards EBM, whether EBM is useful in daily management, whether EBM improves patient care and if EBM places additional demands on overloaded physicians. The last part includes information about barriers to EBM faced by the physicians in obtaining and searching for data: lack of free personal time, limited resources and facilities, no library on the locality and lack of training workshops and courses.

\section{Statistical analysis}

Analysis was carried out to address the following main outcome measures: respondents' attitudes to EBM, their accessibility and interpretation of evidence, their perception of barriers to EBM practice and ways to change from opinion-based to EBM.

All the information gathered was manually reviewed before data entry. The tests of significance used were the chi-squared test and Monte Carlo exact test. SPSS, version 14 was used for the analysis. The significance level was 0.05 (a confidence level of 95\%).

\section{Ethical considerations}

Approval for the research was obtained from the research committee of $\mathrm{Ha}$ mad Medical Corporation. Only PHC physicians who provided consent were included in the study. Confidentiality of data was assured.

\section{Results}

\section{Background characteristics}

Of the 187 of the questionnaires sent out, 182 replies were received, a response rate of $97.3 \%$. The mean age of the responding PHC physicians was 41.4 years. The sex ratio was similar: 48.4\% males and $51.6 \%$ females (Table 1).A high proportion of $\mathrm{PHC}$ physicians practising in Qatar were non-Qatari (74.4\%), with Egyptians forming the largest single nationality (40.7\%).

On average, they had graduated 18.4 years previously and had been practising for 9.2 years in PHC. The time to reach the nearest medical library was less than 30 minutes for $44 \%$ of respondents. Almost one-third of physicians (36.3\%) had more than 50 consultations per shift, and around half of $\mathrm{PHC}$ physicians $(54.9 \%)$ saw $21-50$ patients per shift, while there were no PHC physicians with $<5$ consultations per shift. 


\section{Attitudes towards EBM}

Figure 1 shows the attitudes of the respondents towards EBM. Almost all of them welcomed the current promotion of EBM (98.4\%) and they believed that their colleagues' attitudes were welcoming too. Most agreed that practising EBM improves patient care (96.2\%) and expressed the opinion that research findings were useful in their daily management of patients (90.1\%). The majority (84.6\%) disagreed that EBM was of limited value in primary care but agreed that the adoption of EBM places another demand on already overloaded PHC physicians (56.6\%). Almost three-quarters of them (68.7\%) actively practised EBM.

Table 2 shows the attitudes of the respondents towards EBM according to their characteristics. There was no statistically significant difference between the sexes in attitudes towards EBM. More non-Qatari physicians agreed that EBM was useful in daily management $(92.6 \%)$ than did Qatari physicians (82.6\%) although this was not statistically significant. A few Qatari physicians (10.9\%) believed that EBM was of limited value versus $16.9 \%$ of non-Qataris.

The analysis of attitudes by physician's position found that all the 6 consultants welcomed the current promotion of EBM and believed that their colleagues have positive attitudes towards EBM but also all agreed that EBM created a work overload for physicians (Table 2). Two-thirds (66\%) believed that EBM improved patient care and were actively practising EBM; 83.3\% also agreed that EBM was useful in daily management. All residents and GPs also welcomed the current promotion of EBM and believed that EBM improved patient care, and more than $70 \%$ of them were actively practising EBM. More GPs than residents felt that EBM overloaded physicians. GPs were the physicians who found EBM most useful in daily management (98.1\%).

\begin{tabular}{|c|c|c|}
\hline \multicolumn{3}{|c|}{$\begin{array}{l}\text { Table 1 Demographic characteristics of the sample of primary health care (PHC } \\
\text { physicians }(n=182)\end{array}$} \\
\hline Variable & No. & $\%$ \\
\hline \multicolumn{3}{|l|}{ Sex } \\
\hline Male & 88 & 48.4 \\
\hline Female & 94 & 51.6 \\
\hline \multicolumn{3}{|l|}{ Age (years) } \\
\hline $25-$ & 47 & 25.8 \\
\hline $35-$ & 64 & 35.2 \\
\hline $45-$ & 62 & 34.1 \\
\hline $55-60$ & 9 & 4.9 \\
\hline Mean (SD) & \multicolumn{2}{|c|}{$41.5(8.3)$} \\
\hline \multicolumn{3}{|l|}{ Nationality } \\
\hline Egyptian & 74 & 40.7 \\
\hline Qatari & 46 & 25.3 \\
\hline Sudanese & 12 & 6.6 \\
\hline Palestinian & 12 & 6.6 \\
\hline Syrian & 8 & 4.4 \\
\hline Jordanian & 16 & 8.8 \\
\hline Iraqi & 11 & 6.0 \\
\hline Italian & 2 & 1.1 \\
\hline Pakistani & 1 & 0.5 \\
\hline \multicolumn{3}{|l|}{ Position } \\
\hline Consultant & 6 & 3.3 \\
\hline Specialist & 53 & 29.1 \\
\hline Resident & 18 & 9.9 \\
\hline General practitioner & 103 & 56.6 \\
\hline \multicolumn{3}{|l|}{ Years since graduation } \\
\hline $1-$ & 4 & 2.2 \\
\hline $5-$ & 45 & 24.7 \\
\hline $10-$ & 26 & 14.3 \\
\hline $15-$ & 33 & 18.1 \\
\hline 20- & 36 & 19.8 \\
\hline $25-$ & 32 & 17.6 \\
\hline $30-35$ & 6 & 3.3 \\
\hline Mean (SD) & \multicolumn{2}{|c|}{$18.4(7.9)$} \\
\hline \multicolumn{3}{|l|}{ Years working in PHC } \\
\hline $1-$ & 55 & 30.2 \\
\hline 5- & 58 & 31.9 \\
\hline $10-$ & 29 & 15.9 \\
\hline $15-$ & 19 & 10.5 \\
\hline $20-$ & 18 & 9.9 \\
\hline $25-$ & 2 & 1.1 \\
\hline $30-35$ & 1 & 0.5 \\
\hline Mean (SD) & \multicolumn{2}{|c|}{$9.0(6.7)$} \\
\hline Total & 182 & 100.0 \\
\hline
\end{tabular}

$S D=$ standard deviation. 


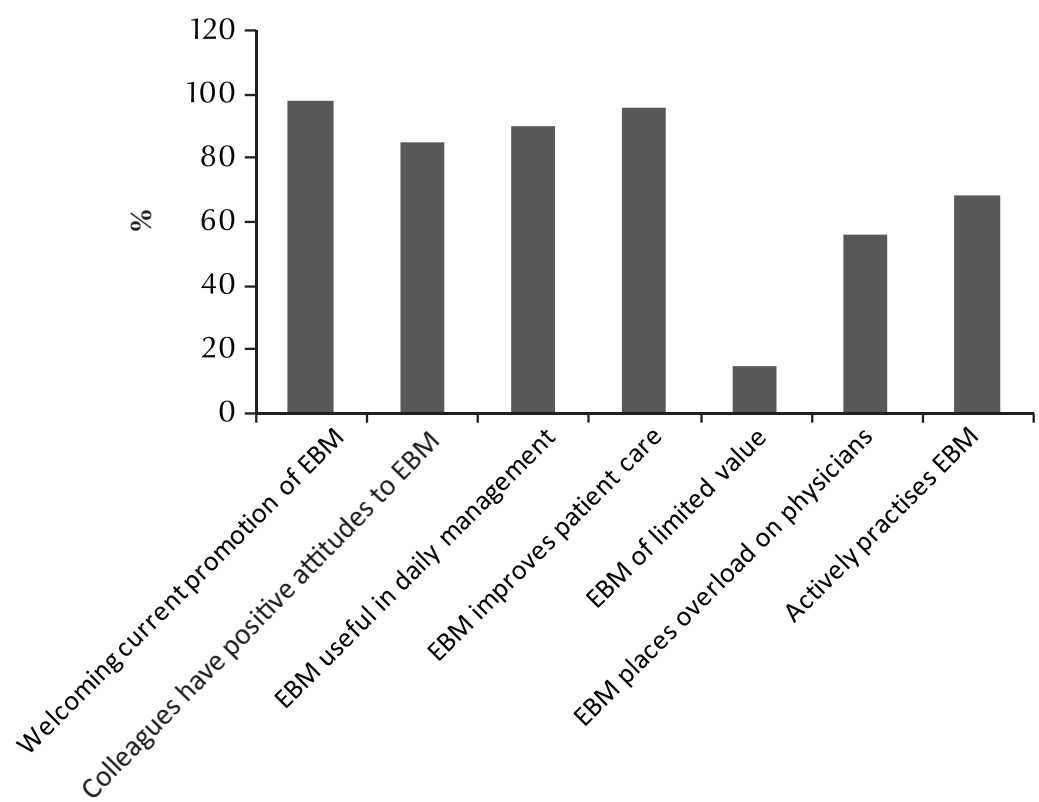

Figure 1 Attitudes towards evidence-based medicine (EBM) of primary health care physicians $(\boldsymbol{n}=182)$

Analysis of the time since graduation shows that the highest proportion of PHC physicians who actively practised EBM had 21-30 years since graduation (83.3\%), while the lowest rate (33.0\%) was among those who had graduated $31-40$ years ago (Table 2 ).

Physicians who saw 5-20 patients per day were the most likely to practise EBM (81.2\%), welcome current promotion of EBM, find it useful in daily management and believe that it improved patient care $(100 \%)$ (Table 2).

Table 2 also shows that the closer they were to a library the more likely it was that physicians considered EBM useful in daily management, believed that it improved patient care and practised EBM

\section{Practice of EBM}

The major perceived barriers to practising EBM in primary care was lack of free personal time (75.3\%), limited resources and facilities (62.6\%), no library on the locality $(61.0 \%)$ and lack of training workshops and courses (61.0\%).

Table 3 shows that approximately $52.7 \%$ of the PHC physicians were aware of the American College of Physicians' Journal Club and $40.7 \%$ were aware of EBM websites. Only 26.4\% and $17.0 \%$ were aware of the Cochrane Database of Systematic Reviews and Best Evidence Review, respectively. About $19.2 \%$ were aware of the Effective Health Care Bulletin.

Most of the physicians had access to the Internet (92.2\%) but this was usually at home. More than one-third of the physicians (35.0\%) were reading journals on demand and another one-third (34.6\%) were reading them regularly; $28.0 \%$ read them occasionally and only a few physicians reported never reading journals (2.2\%). Table 3 shows that the most commonly read journal was the British Medical Journal (69.2\%), followed by the Qatar Medical Journal (40.0\%). Middle East Medical Journal of Family Medicine, The Practitioner and Medicine Digest were the least accessed.

Figure 2 shows that the respondents were comfortable with most of the technical terms in EBM (risk factors, relative risk, sensitivity, specificity, clinical effect, meta-analysis, number needed to treat and risk reduction), and a sizeable proportion felt that they could explain them to others. Attributable risk, likelihood ratio and odds ratio were the terms that were poorly understood.

Table 4 shows the frequency of reading journals by physicians' characteristics. The highest proportion of physicians regularly reading journals were those aged 45-54 years, with 20-24 years since graduation, Syrian nationality, in a consultant position and working 15-20 years in PHC.

\section{Discussion}

The welcoming attitudes of $\mathrm{PHC}$ physicians in Qatar towards EBM are similar to those in Saudi Arabia $[16,17]$, UK [15] and Australia [18]. Nowadays, there is a worldwide plethora of books, workshops and courses on how to practise and teach EBM. Electronic and online databases of systematic reviews and summaries of evidence are increasingly becoming available for PHC physicians [19-21]. The PHC physicians in Doha had a high level of awareness of these resources compared with those practising in the Riyadh region of Saudi Arabia [16]. In spite of that, they mostly did not make use of them in clinical 


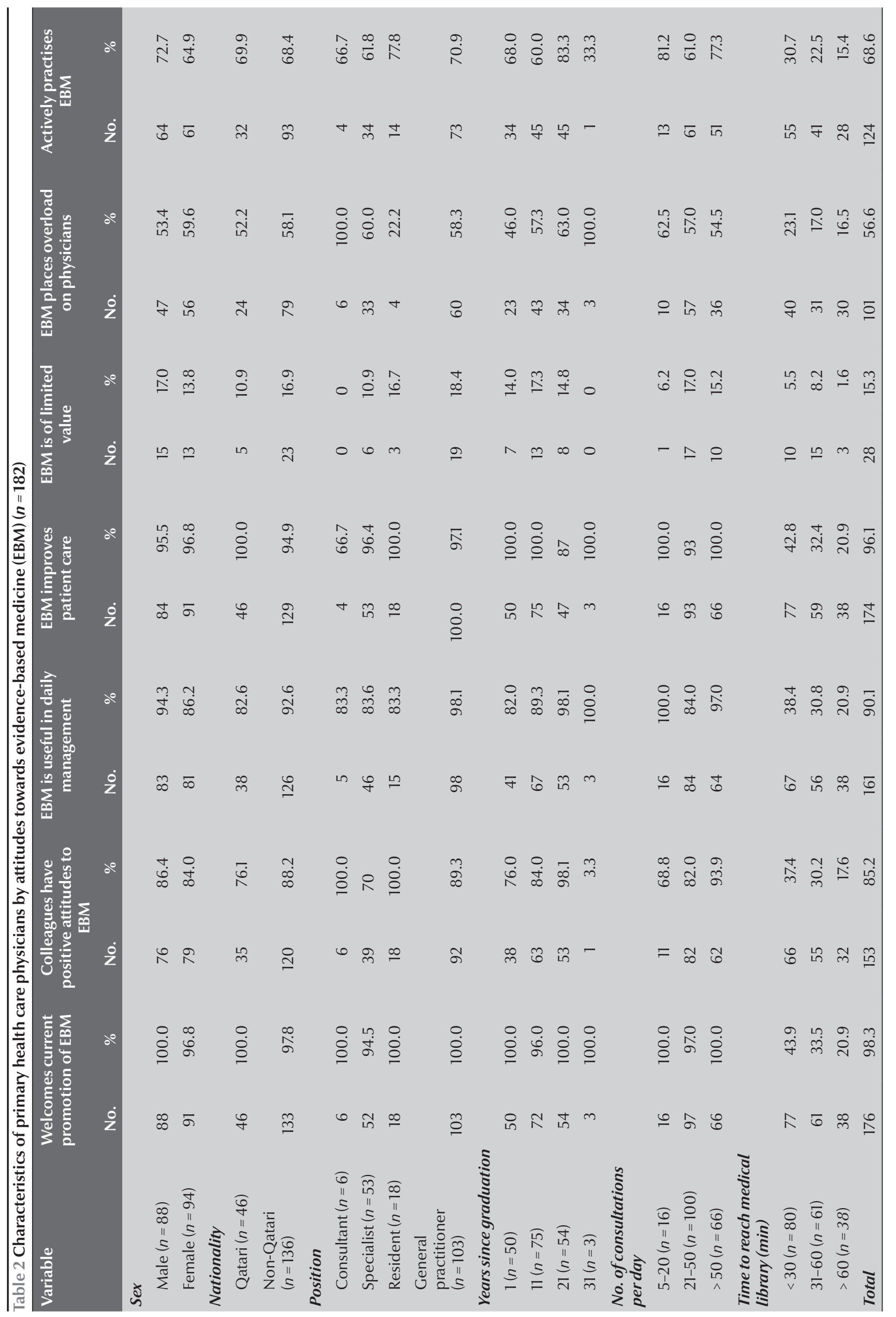




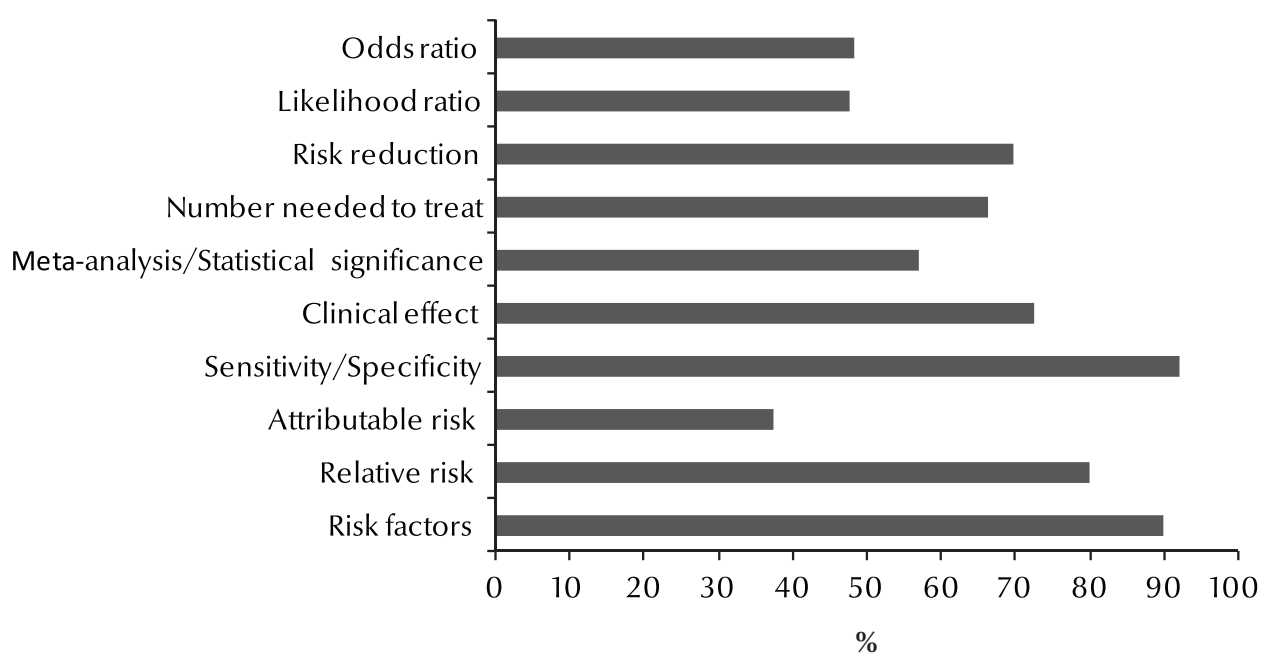

Figure 2 Understanding of technical terms used in evidence-based medicine (EBM) of primary health care physicians $(n=182)$

decision-making. Although EBM encourages the use of primary research studies, evidence-based clinical practice guidelines and systematic overviews to inform treatment decisions [22], recent surveys have suggested that most physicians still rely heavily on the opinion of colleagues or consultants when making these decisions [23-25].

The technical terms commonly used in EBM are the key element in practising EBM. Variation between understanding of medical terms among PHC physicians could hinder their interpretation and make dissemination of evidence more difficult.

Lack of EBM training courses and workshops was also seen as a barrier to practicing EBM by physicians in Doha. The most appropriate way to move from opinion-based medicine to EBM is through focusing on training in the critical appraisal of research. It was found in the Riyadh study that most of the respondents (42.6\%) thought that the best way to move from opinionbased medicine to EBM was by learning the skills of EBM, while (37.0\%)

\begin{tabular}{lcc}
\hline $\begin{array}{l}\text { Table } 3 \text { Ever-use of resources for clinical decision-making and journals usually } \\
\text { read by primary health care physicians }(\boldsymbol{n}=\mathbf{1 8 2})\end{array}$ & No. $(\boldsymbol{n}=\mathbf{1 8 2})$ & $\%$ \\
\hline Variable & & \\
Ever-used resource & 96 & 52.7 \\
American College of Physicians journal club & 48 & 26.4 \\
Cochrane Database of Systemic Reviews & 35 & 19.2 \\
Effective Health Care Bulletin & 31 & 17.0 \\
Ovid's Evidence-Based Medicine Review & 17 & 9.3 \\
Best Evidence Review & 74 & 40.7 \\
Other EBM websites & & \\
Journals usually read & 126 & 69.2 \\
British Medical Journal & 73 & 40.0 \\
Qatar Medical Journal & 54 & 29.7 \\
Middle East Medical Journal of Family Medicine & 33 & 18.1 \\
The Practitioner & 34 & 18.7 \\
Medicine Digest & 67 & 36.8 \\
Other
\end{tabular}

thought it should be by using evidencebased guidelines or protocols [16]. In contrast the largest proportion of $\mathrm{PHC}$ physicians in McColl's study in the UK (57\%) thought that the most appropriate way to apply EBM was by using evidence-based guidelines or protocols, while $37 \%$ thought it should be by seeking and applying evidence-based summaries and only (5\%) by identifying and appraising the primary literature or systematic reviews [15].

The most commonly read journals among respondents were the British Medical Journal (by 69.2\%) and Qatar Medical Journal (40.0\%), which is logical because both journals are widely available. These journals can be helpful for practising EBM. In the Asir region study the free-of-charge non-peer-reviewed journals sponsored by pharmaceutical companies The Practitioner and Medicine Digest were the most regularly read (by $20.2 \%$ and $18.8 \%$ of physicians respectively) [17], while in Riyadh region, The Practitioner and Medicine Digest were the most regularly read journals (by $29.9 \%$ and $24.0 \%$ respectively) [16].

A considerable proportion of respondents perceived personal lack of time $(75.3 \%)$ as a major barrier to practising EBM. In McColl's study, lack of personal time was also the main perceived barrier to practising EBM 


\begin{tabular}{|c|c|c|c|c|c|c|c|c|}
\hline \multirow[t]{3}{*}{ Variable } & \multicolumn{8}{|c|}{ Frequency of reading journals } \\
\hline & \multicolumn{2}{|c|}{ Never } & \multicolumn{2}{|c|}{ Occasionally } & \multicolumn{2}{|c|}{ On demand } & \multicolumn{2}{|c|}{ Regularly } \\
\hline & No. & $\%$ & No. & $\%$ & No. & $\%$ & No. & $\%$ \\
\hline \multicolumn{9}{|l|}{ Sex } \\
\hline Male & 2 & 2.3 & 18 & 20.5 & 37 & 42.0 & 31 & 35.2 \\
\hline Female & 2 & 2.1 & 33 & 35.1 & 27 & 28.7 & 32 & 34.0 \\
\hline \multicolumn{9}{|l|}{ Age (years) } \\
\hline $25-$ & 3 & 6.4 & 23 & 48.9 & 10 & 21.3 & 11 & 23.4 \\
\hline $35-$ & 1 & 1.6 & 12 & 18.8 & 29 & 45.3 & 22 & 34.4 \\
\hline $45-$ & 0 & 0.0 & 16 & 25.8 & 19 & 30.6 & 27 & 43.5 \\
\hline $55-60$ & 0 & 0.0 & 0 & 0.0 & 6 & 66.7 & 3 & 33.3 \\
\hline \multicolumn{9}{|l|}{ Nationality } \\
\hline Egyptian & 1 & 1.4 & 22 & 29.7 & 25 & 33.8 & 26 & 35.1 \\
\hline Qatari & 2 & 4.3 & 14 & 30.4 & 16 & 34.8 & 14 & 30.4 \\
\hline Sudanese & 0 & 0.0 & 6 & 50.0 & 1 & 8.3 & 5 & 41.7 \\
\hline Palestinian & 0 & 0.0 & 1 & 8.3 & 6 & 50.0 & 5 & 41.7 \\
\hline Syrian & 0 & 0.0 & 1 & 12.5 & 2 & 25.0 & 5 & 62.5 \\
\hline Jordanian & 0 & 0.0 & 5 & 31.3 & 9 & 56.3 & 2 & 12.5 \\
\hline Iraqi & 1 & 9.1 & 0 & 0.0 & 4 & 36.4 & 6 & 54.5 \\
\hline Italian & 0 & 0.0 & 1 & 50.0 & 1 & 50.0 & 0 & 0.0 \\
\hline Pakistani & 0 & 0.0 & 1 & 100.0 & 0 & 0.0 & 0 & 0.0 \\
\hline \multicolumn{9}{|l|}{ Position } \\
\hline Consultant & 0 & 0.0 & 1 & 16.7 & 2 & 33.3 & 3 & 50.0 \\
\hline Specialist & 3 & 5.7 & 14 & 26.4 & 14 & 26.4 & 24 & 45.3 \\
\hline Resident & 0 & 0.0 & 8 & 44.4 & 5 & 27.8 & 5 & 27.8 \\
\hline General practitioner & 1 & 1.0 & 28 & 26.2 & 43 & 41.7 & 31 & 30.1 \\
\hline \multicolumn{9}{|l|}{ Years since graduation } \\
\hline $1-$ & 1 & 25.0 & 0 & 0.0 & 2 & 50.0 & 1 & 25.0 \\
\hline $5-$ & 2 & 4.4 & 23 & 51.1 & 8 & 17.8 & 12 & 26.7 \\
\hline $10-$ & 0 & 0.0 & 9 & 34.6 & 14 & 53.8 & 3 & 11.5 \\
\hline $15-$ & 0 & 0.0 & 10 & 30.3 & 15 & 45.5 & 8 & 24.2 \\
\hline $20-$ & 1 & 2.8 & 4 & 11.1 & 9 & 25.0 & 22 & 61.1 \\
\hline $25-$ & 0 & 0.0 & 5 & 15.6 & 11 & 34.4 & 16 & 50.0 \\
\hline $30-35$ & 0 & 0.0 & 0 & 0.0 & 2 & 33.3 & 1 & 16.7 \\
\hline \multicolumn{9}{|l|}{ Years working in PHC } \\
\hline $1-$ & 3 & 5.5 & 24 & 34.6 & 15 & 27.3 & 13 & 23.6 \\
\hline 5- & 0 & 0.0 & 19 & 32.8 & 23 & 39.7 & 16 & 27.6 \\
\hline $10-$ & 1 & 3.4 & 4 & 13.8 & 8 & 27.6 & 13 & 44.8 \\
\hline $15-$ & 0 & 0.0 & 1 & 5.3 & 5 & 26.3 & 13 & 68.4 \\
\hline 20- & 0 & 0.0 & 3 & 16.7 & 12 & 66.7 & 3 & 16.7 \\
\hline $25-$ & 0 & 0.0 & 0 & 0.0 & 1 & 50.0 & 1 & 50.0 \\
\hline $30-35$ & 0 & 0.0 & 0 & 0.0 & 0 & 0.0 & 1 & 100.0 \\
\hline
\end{tabular}

for $71 \%$ of GPs [15]. If the current best evidence is not considered, the clinical practice of PHC physicians is possibly at risk of becoming out of date, to the detriment of patients [20,21]. Dawes' study suggested that there are ways of increasing the time available for practising EBM [3]. Physicians' time could be spent more efficiently by changing the emphasis of postgraduate education away from lectures towards training in accessing and interpreting evidence and then spending time putting these skills into practice $[3,26]$. While it has been said that GPs need to come to grips with 
19 original articles per day, 365 days per year if they want to keep abreast of their field [27], Dawes suggested that a GP who spent only 1 hour per week searching for and reading articles would make huge strides in implementing evidence [3].

However, our physicians noted the absence of a library in the locality, the long travel distances to reach the nearest library and limited resources and facilities. Limited resources are in the form of lack of computers in addition to deficient Internet access in the workplace. There was limited access to online journals because few physicians had access to journal subscriptions. Lack of availability of books, especially up-to-date ones, in many of the specialties were also seen as a major barrier. Most of the respondents in our study had access to the Internet, but for the majority this was at home not the workplace. This may explain the low rate of practising EBM. The Internet promotes the practice of EBM by facilitating the generation, synthesis, dissemination and exchange of research evidence [28]. Giving physicians cheap, fast and efficient access to up-to-date, valid, relevant and "just-in-time" knowledge is essential.

It has been shown that GPs trained in the skills of critical appraisal were more likely to translate evidence from systematic reviews into practice [29]. With more systematic reviews becoming available, there are more resources to support changes in clinical behaviour. Yet, even with well-developed evidencebased guidelines, tensions and contradictions impede their translation into policy and practice [30]. Research has recommended that combined activities in EBM were in general better than single interventions, and that changes in practice occurred more frequently when barriers to change were addressed and resources deployed to help learners [31]. It has been suggested that if clinical practice guidelines are incorporated into electronic medical record systems to assist clinical decision-making at the point of care, it may greatly enhance the delivery of evidence-based clinical practice [32].

Furtherfollow-up research is needed on the practice and implementation of EBM at PHC level in our region. Although our study was preliminary, it has provided useful findings that have motivated us to improve our teaching on EBM for PHC physicians in the near future.

This study has limitations that must be mentioned. As with any survey, there is the possibility of a social response bias. Physicians may tend to over-report their use of journals and textbooks and underreport their reliance on human sources. Thus, our finding of a high self-reported reliance on colleagues and expert opinion may even be an underestimate of the picture in actual practice. Nevertheless the response rate to our survey was high at $97.3 \%$, higher than the figure of $86 \%$ reported in studies in Asir [17] and Riyadh [16].

\section{Conclusions}

The results of this study highlight the need to stimulate the use of EBM among physicians working in primary care. Action is needed to improve access to and utilization of Internet-based evidence databases. Addressing and overcoming the barriers of practising EBM is crucial.

\section{References}

1. Rosenberg W, Donald A. Evidence-based medicine: an approach to clinical problem solving. British Medical Journal, 1995, 310:1122-1126.

2. Ridsdale L. Evidence-based learning for general practice. British Journal of General Practice, 1996, 46:503-504.

3. Dawes M. On the need for evidence-based general and family practice. Evidence-based Medicine, 1996, 1:68-69.

4. Baker R. North of England evidence based guidelines development project: Summary version of evidence based guideline for the primary care management of stable angina. British Medical Journal, 1996, 312:827-832.

5. North of England Asthma Guideline Development Group. North of England evidence based guidelines development project: summary version of evidence based guideline for the primary care management of asthma in adults. British Medical Journal, 1996, 312:762-766.

6. Eccles M. North of England evidence based guidelines development project: methods of guideline development. British Medical Journal, 1996, 312:760-762.

7. McColl A. Performance indicators for primary care groups: an evidence based approach. British Medical Journal, 1998, 317:1354-1360.
8. Gill P. Evidence based general practice: a retrospective study of interventions in one training practice. British Medical Journal, 1996, 312:819-821.

9. Roper WL. Effectiveness in health care: an initiative to evaluate and improve medical practice. New England Journal of Medicine, 1988, 319:1197-1202.

10. Sackett DL. Clinical epidemiology: a basic science for clinical medicine, 2nd ed. London, Little Brown, 1991:305-333.

11. Greenhalgh T. Is my practice evidence-based? British Medical Journal, 1994, 309:597-599.

12. Davis DA. Changing physician performance: a systematic review of the effect of continuing medical educational strategies. Journal of the American Medical Association, 1995, 274:700-705.

13. Dowie J. 'Evidence-based', 'cost-effective' and 'performancedriven' medicine: decision analysis based medical decisionmaking is the pre-requisite. Journal of Health Services Research and Policy, 1996:1:104-113.

14. Silagy C, Haines A, eds. Evidence based practice in primary care. London, British Medical Journal Publishing Group, 1998. 
15. McColl A. General practitioners' perceptions of the route to evidence based medicine: a questionnaire survey. British Medical Journal, 1998, 316:361-365.

16. Al-Ansary LA, Khoja TA. The place of evidence based medicine among primary health care physicians in Riyadh region, Saudi Arabia. Family Practice, 2002, 19:537-42

17. Al-Ansary LA, Khoja TA. Attitudes to evidence-based medicine of primary care physicians in Asir region, Saudi Arabia. Eastern Mediterranean Health Journal, 2007, 13:2.

18. Mary J, Piterman L. The attitudes of Australian GPs to evidencebased medicine: a focus group study. Family Practice, 1999, 16:627-632.

19. Essential Evidence Plus [online database] (http://www.essentialevidenceplus.com/, accessed 28 September 2010).

20. Sackett DL. Evidence-based medicine: how to practice and teach $E B M, 2$ nd ed. London, Churchill Livingstone, 2000.

21. Yamey G. Subjectivity can be inhumane. Western Journal of Medicine, 2000, 173:143.

22. Chalmers I, Dickersin K, Chalmers TC. Getting to grips with Archie Cochrane's agenda. British Medical Journal, 1992, 305:786-787.

23. Hayward RSA et al. Canadian physicians' attitudes about and preferences regarding clinical practice guidelines. Canadian Medical Association Journal, 1997, 156:1715-1723.
24. McAlister FA. Influencing practice patterns in hypertension. Canadian Medical Association Journal, 1997, 157:1348-1349.

25. Tunis SR, Hayward RSA, Wilson MC. Internists' attitudes about clinical practice guidelines. Annals of Internal Medicine, 1994, 120:956-963.

26. Davidoff F et al. Evidence based medicine: a new journal to help doctors identify the information they need. British Medical Journal, 1995, 310:1085-1086.

27. Sackett DL. ...So little time, and.... Evidence-based Medicine, 1997, 2:39.

28. Jadad AR. The internet and evidence-based decision-making: a need synergy for efficient knowledge management in health care. Canadian Medical Association Journal, 2000, 162:362365.

29. Kidd MR, Mazza D. Clinical practice guidelines and the computer on your desk. Medical Journal of Australia, 2000, 173:373375 .

30. Doust JA, Silagy CA. Applying the results of a systematic review in general practice. Medical Journal of Australia, 2000, 172:153-156.

31. Swinglehurst D, Pierce M. Questioning in general practice-a tool for change. British Journal of General Practice, 2000, 50:747-750.

32. Hirst G, Ward J. Clinical practice guidelines: reality bites. Medical Journal of Australia, 2000, 172:287-291.

\section{WHO Research for Health Strategy}

In May 2010 the first organization-wide strategy on research was approved by the World Health Assembly. The strategy - Research for Health - defines a common framework for how research is approached in WHO and the role WHO is taking in global health research.

The strategy has five main goals:

- Capacity - building capacity to strengthen health research systems.

- Priorities - supporting the setting of research priorities that meet health needs particularly in low- and middle-income countries.

- Standards - creating an environment to create good research practice and enable the greater sharing of research evidence, tools and materials.

- Translation - ensuring quality evidence is turned into products and policy.

- Organization - action to strengthen the research culture within WHO and improve the management and coordination of WHO research activities. 